\title{
ACOUPLETTES, BOUDINE, MARGOULETTE ET AUTRES UNITÉS RÉGIONALES POUR BROSSER LE PORTRAIT PHYSIQUE ET STIGMATISER AUTRUI À L'ARDENNAISE ${ }^{1}$
}

\author{
Tatiana RETINSKAYA \\ Université d'État d'Orel Tourgueniev
}

\begin{abstract}
En): This article focuses on the study of regional lexical funds that relate to the human body. It is devoted especially to the analysis of the paradigmatic and syntagmatic links of Ardennisms aimed at designating physical peculiarities, including bodily disability. The body of examples, consisting of written documents and oral statements gathered by means of field studies, reflects an obviously fruitful ability of the regiolectophone. It will allow us to examine the names of the external and internal parts of the body used by the speakers, such as parts of the head, physique, birth defects, habits and those used in situations related to the need to describe the height, the state of health or to explain the causes that disrupt the functioning of this or that organ; without forgetting the use of this stratum of the specific vocabulary used for self-deprecation as well as for the devaluation of others. Particular attention is paid to the classification of the relevant regional elements specific to the child language. Thus, we hope to highlight an aspect of the richness and specificity, if not originality, of the Ardennes regiolect in its contemporaneity.
\end{abstract}

Keywords (En): human body; physical particularities; Ardennes regiolect; Ardennism; stigmatization of others; tropological space

Mots-clés (Fr) : corps humain ; particularités physiques ; parler des Ardennes ; ardennisme ; stigmatisation d'autrui ; espace tropologique

DOI : $10.32725 /$ eer.2021.002

L'invective, la critique acerbe, le jugement d'autrui, la moquerie puisent dans le vivier du vocabulaire régional des traits redoutables, beaucoup plus efficaces qu'un développement circonstancié.

Michel TAMINE

\section{Introduction}

Comme l'épigraphe choisie pour la présente contribution le laisse entendre, la fonction de désignation du langage régional est inséparable de celle d'évaluation. Les observations de Michel TAMINE (2006: 8) sont confirmées lors de l'analyse des fonds lexicaux du parler des Ardennes qui se rapportent au corps humain. Si l'on relève les multiples vecteurs de la description de l'idiome régional, un constat incontestable s'impose : «[...] le lexique est le domaine qui offre le champ d'observation le plus vaste à l'étude de la variation » (RÉZEAU, 2001 : 10), sans omettre bien évidemment la variété des sources pour obtenir un corpus représentatif.

\footnotetext{
${ }^{1}$ Cet article synthétise les résultats intermédiaires de la réalisation du projet de l'étude des parlers régionaux mené dans le cadre du Laboratoire Description des variétés sociales et territoriales du français de l'Université d'État d'Orel Tourgueniev.
} 
Celui-ci est constitué d'attestations écrites - ouvrages lexicographiques (BÉSÈMEPIA, 2011 ; COLIN, 1957 ; CORDIER, 1988 ; HUART, 1988; TAMINE, 2006 ; VAUCHELET, 1979), atlas linguistiques (BOURCELOT, 1966; BOURCELOT \& TAMINE, 2012), articles particuliers (CLERC, 1988-1989 ; LAUBY \& TAMINE, 1987), documents d'archives ${ }^{2}$ (Patois de Guignicourt, Faissault et Champigneul-surVence, 1929 ; CORDIER, 1975), œuvres des auteurs du terroir (BRAIBANT, 1933 ; PETITFAUX, 1962 ; ROGISSART, 1994), ainsi que de témoignages oraux par le moyen d'enquêtes sur le terrain.

Notre article présentera, d'une part, les résultats de la classification des parties du corps, ou somatismes, propres au parler ardennais, d'autre part, l'analyse des liens paradigmatiques et syntagmatiques des ardennismes visant à désigner les particularités physiques, y compris la disgrâce corporelle. En effet, le corpus d'exemples reflète une aptitude assurément féconde du régiolectophone. Il nous a permis d'examiner les dénominations des parties du corps utilisées par les locuteurs, comme les détails de la tête, la corpulence, les malformations congénitales, les habitudes, et celles employées dans les situations liées à la nécessité de décrire la taille, l'état de santé ou d'expliquer les causes qui perturbent le fonctionnement de tel ou tel organe; sans oublier le recours à cette strate du vocabulaire spécifique servant à stigmatiser les voisins. Notre objectif est également d'inventorier les éléments régionaux en question propres au langage enfantin.

Nous espérons ainsi pouvoir mettre en lumière un aspect de la richesse et de la spécificité, si ce n'est de l'originalité, du régiolecte ardennais dans sa contemporanéité.

\section{Regroupement des matériaux lexicaux relatifs au corps}

\subsection{Centres d'intérêt inventoriés par Henri BourCELOT}

L'auteur des quatre volumes de l'Atlas linguistique et ethnographique de la Champagne et de la Brie qui avait réussi à « recueillir les trois cent mille fiches linguistiques, ethnographiques et folkloriques» (BOURCELOT \& TAMINE, 2012 : 229) considérait le «questionnaire par centres d'intérêt» comme "guide de l'enquête » (BOURCELOT \& TAMINE, 2012 : 135). Il n'est guère étonnant que les questions de ce questionnaire aient visé à obtenir des unités régionales désignant le portait physique de l'homme comme individu et comme membre d'une collectivité. Cette couche importante du parler ardennais a été organisée par le biais du centre d'intérêt du questionnaire intitulé Anatomie qui comprenait quatre grandes composantes :

1)Tête, cheveux, yeux.

2) Nez, gorge, oreilles.

3) Bras, jambes.

4) Viscères, os.

\footnotetext{
${ }^{2}$ L'auteure remercie l'équipe des Archives départementales des Ardennes qui lui a facilité sa recherche sur place et lui a permis de s'immerger dans des documents rares et précieux.
} 
Par ailleurs, les grandes parties du centre d'intérêt relevé comportent 113 questions conçues pour indiquer les traits physiques d'une personne. Citons, à titre d'exemple, les éléments du questionnaire servant à décrire les yeux :

1) Les cils.

2) La lumière fait ciller.

3) Les sourcils.

4) L'œil droit.

5) L'œil gauche.

6) Les deux yeux.

7) La pupille de l'œil.

8) Un orgelet.

9) Il a les yeux chassieux.

10) Regarder.

11) Regarde bien !

12) Écarquiller les yeux (regarder curieusement en ouvrant de grands yeux).

13) Regarder bêtement.

14) Il est borgne.

15) Il est aveugle.

16) Il louche.

17) Un louchon.

18) Je suis ébloui : j’ai la vue troublée.

19) Des éblouissements (bleues vues, bluettes) (BOURCELOT \& TAMINE, 2012 : 248-265).

Ce mode pertinent de distribution des éléments du vocabulaire est devenu l'une des premières pistes à explorer pour la systématisation et la présentation de l'univers conceptuel du corps humain. C'est pourquoi cette approche de collecte des lexèmes régionaux a servi comme axe pour nos enquêtes de terrain.

\subsection{Classification des champs conceptuels}

Le corpus de somatismes que nous avons recueilli contient 317 unités. Les données et les métadonnées de l'inventaire des noms évoquant le physique d'une personne permettent d'effectuer une classification de divers champs conceptuels. Ainsi, les vocables ardennais se répartissent dans de nombreux groupes de dénomination. Voici les exemples les plus représentatifs ${ }^{3}$ :

1) tête, visage : gonelle - margoulette;

2) nuque : aunette - (h) ânette - hênette;

3) bouche : goûle ;

4) gosier : gosillon - gosillot-goulette;

5) pomme d'Adam : gosillon;

6) œil : pèrouya, ziu ;

\footnotetext{
${ }^{3}$ Comme le montrent ces exemples, certains ardennismes présentent une « déformation » phonétique par rapport à leurs équivalents en français standard. Pour toutes les étymologies des régionalismes étudiés dans l'article, v. l'ouvrage lexicographique de Michel TAMINE (2006).
} 
7) dent : $d a$;

8) nez: nèye ;

9) oreille : oureille;

10) main : paouine (pawène) ;

11) jambe : dègrè-jame ;

12) pied : pie ;

13) genou : $g(e) r o n$;

14) orteil : dôye;

15) petit orteil : doyette;

16) os: o(u)che;

17) ossement : och'ma;

18) arête du tibia : grè ;

19) tendon : nerf;

20) échine : housse du dos ;

21) sein : gougoute ;

22) ventre : berdouille - quiqui - vat(r)e - vente;

23) nombril : boudette-boudine-boudr(o) ûle-boutr(o)ûle;

24) raie de fesses : rôye ;

25 ) testicules : baloces (balosses) - bobines - bûrettes - prunes - robignoles ;

26) sexe de femme : craîlle - craîllette.

Au-delà des noms de parties externes et internes du corps humain, le corpus étudié comprend l'ensemble des unités caractérisant des particularités physiques. Il en est ainsi, en premier chef, de la désignation des différences de constitution corporelle :

1) une personne robuste : corporé - gadru;

2) une personne de petite taille : nainjandio - nine ;

3) une personne chétive : chècreux - chîcreux - neuquet-raboset-rachichisèqueron ${ }^{4}$

4) une personne obèse : moyon - patapouf;

5) une personne voûtée : hottu;

6) une personne au ventre proéminent : pansu-pointu.

Par ailleurs, le corpus met également en lumière des régionalismes désignant des aspects physionomiques dus à la vieillesse ou à la maladie :

1) une personne amoindrie par l'âge : caduc ;

2) une personne affaiblie par la maladie : affligé - arrangé - saqué ;

3) une personne amaigrie : ramaigri;

4) une personne qui sort de la maladie : lâdre.

De plus, il est important de mentionner la présence de lexèmes régionaux qui englobent simultanément deux ou plusieurs traits caractéristiques d'un individu. Il en est ainsi de hoche-bobot ('personne de petite taille et fluette'), de posson potiquet ('personne de petite taille et grosse'), de rabo(u)zet ('enfant frêle et menu'),

\footnotetext{
${ }^{4}$ « Tout ce qui était petit, menu, chétif ou malingre était mal vu, déprécié » (BÉSĖME-PIA, 2011 : 49).
} 
de nervaillon - nervillon ('personne fluette, chétive et nerveuse') ou encore de nounousse ('homme gros, gentil et jovial').

Parmi ceux-ci, le lexème rètu est un cas particulier. En effet, cet ardennisme, « éventuellement précisé par un adverbe, déjà ou encore, s'applique aussi bien au jeune enfant qu'au vieillard; il implique la bonne santé physique, l'aptitude à résister aux maladies, la vivacité de l'esprit » (TAMINE, $2006: 6)^{5}$. Nos relevés ont bien confirmé cet emploi. Ainsi, nous avons pu relever à Librecy, s'agissant d'un vieillard : «- T'en fais pas, il est encore rètu. » Et à Signy l'Abbaye, à propos d'un jeune enfant, et dans un tout autre contexte : «-Oh, elle, pas de problème, elle est bien rètue, elle court partout. "

En outre, il peut arriver que certaines unités locales soient intégrées dans le processus de dénomination des termes d'injure, comme tôye ('grosse femme'), nabochet - nabochon ('homme de petite taille'). Toutefois, la nature des mots obscènes appartenant au substrat régional est assez particulière, comme le précise BÉSĖME-PIA, lorsqu'elle évoque le statut des offenses typiques aux régiolectophones :

Beaucoup de mots ou expressions auxquels on attribuerait aujourd'hui un sens péjoratif ou vulgaire, voire même insultant, étaient employés et considérés couramment comme gentils, sympathiques ; même certaines injures pouvaient être gentilles : tout dépendait des circonstances et surtout du ton sur lequel elles étaient dites. [...] On appelait certaines personnes plus ou moins fortes « Ma gros » ou « Ma grosse » sans aucune idée péjorative ou moqueuse, au contraire. [...] On aurait tendance aujourd'hui à s'offusquer trop facilement de certaines appellations dont on prend un malin plaisir à faire dévier la signification primitive vers un sens défavorable, voire insultant. (BÉSÈME-PIA, $2011:$ 19)

Quoi qu'il en soit, la stigmatisation d'autrui s'effectue par le biais de sa disgrâce physique. En effet :

«Les imperfections physiques étaient mal supportées autrefois. On affublait des qualificatifs d'une raillerie féroce tous ceux qui n'étaient pas conformes à la règle. Autrefois, c'était parce que ces gens ne pouvaient pas travailler et se trouvaient à la charge des autres. Il reste encore aujourd'hui une certaine hostilité envers ceux qui ne sont pas comme tout le monde : c'est une sorte de réflexe avec les générations précédentes » (BOURCELOT \& TAMINE, 2012 : 104).

Sont ainsi présentes dans cette catégorie les malformations congénitales, telles bancalot - clibaucheux - tantôt il buque, tantôt il buquelle - tantôt il dauve, tantôt il dauville ('boiteux'), la douze dôye (< dôye 'orteil'), pîe boquet ('personne atteinte d'une malformation congénitale du pied') ..., sans oublier le recours à la strate du vocabulaire spécifique servant à pourvoir des voisins d'une caractéristique particulière de leurs corps et fournissant des sobriquets individuels : La Boudine ('femme énorme', < boudine 'nombril', 'ventre'), El Fiderbe ('homme grand et maigre', < le fil d'herbe). Cette caractéristique se justifie par le fait que :

\footnotetext{
${ }^{5}$ Rétu [sic] se trouve logiquement en picard contemporain, mais semble avoir le sens moins extensif de « vif», v. Glossaire des mots picards, termes et expressions encore en usage dans le Vermandois (1953-1954: 64).
} 
« [...] les particularités physiques, les plus immédiatement perceptibles, fournissent [...] un contingent appréciable de formes ; mais le village est un lieu clos, à l'intérieur duquel s'exaspère de façon quasi pathologique l'observation permanente et réciproque des individus. [...] Nombre de sobriquets résultent donc de la surveillance sociale et l'observation psychologique : on trouve sans doute dans cette catégorie les plus caustiques, encore que la méchanceté pure soit pratiquement exclue [...]»(LAUBY \& TAMINE, $1987: 36-39)$.

Qui plus est, dans le français régional des Ardennes, on découvre une couche lexicale destinée à caractériser les transformations du corps : prise - comme çaaprèsurée - aperzurée ('enceinte') et les parties du corps abimées à cause d'une blessure, d'une maladie, d'une piqûre ou d'une morsure, comme dent de l'œil ('une dent dont le nerf est réputé rejoindre le nerf optique'), dôsse ('boursouflure provoquée par une piqûre d'insecte ou d'ortie'), gougouille ('adénite'), graffiné ('égratigné'), maque ('trace de coup sur la peau'), mau de Saint-Min ('lèvres couvertes de croûtes'), mais aussi les marques laissées sur la peau par la maladie : mouflettes ('rougeole'), poques volantes ('varicelle'), poquettes ('variole'), résipel ('érysipèle'), tigne ('teigne'), les traits visibles sur le visage : brans de judas ('taches de rousseur'), tachu ('homme marqué de taches naturelles sur le visage'), les dommages de la peau par suite du froid, de la chaleur ou de l'âge : èbisé ('gercé par le froid'), (é)pinçons - pînchons - piquette ('onglée'), raguerzillé - raguerzilliee ('ratatiné par suite du froid, de la chaleur ou de l'âge'), ou encore les causes qui perturbent le fonctionnement de tel ou tel organe : papillule ('cil recourbé qui provoque une gêne sur l'œil'), l'aspect corporel: trouillon ('personne sale qui manque de propreté corporelle') et les habitudes : pieds déchaux ('pieds nus').

De nombreux lexèmes locaux se sont aussi imposés au langage enfantin. Citons, entre autres, bibique - didique ('dent'), boudette ('nombril'), boudine ('ventre'), carreau ('gros ventre chez les enfants'), chiquette ('sexe d'un petit garçon'), chounette ('sexe de petite fille'), coûches ('fesses'), gnouf ('pourtour sale de la bouche d'un enfant qui vient de manger'), minotte ('main'), quiqui ('gorge') et yin (-)yin ('auriculaire').

\subsection{Composants de l'espace tropologique}

Il s'avère que le potentiel sémantique du vocabulaire en question est assez important ; il est mesuré en fonction de marqueurs tels que l'usage sans faux fuyants du mot régional, l'expressivité et la perception visuelle. À cet endroit, le terme « traits saillants » proposé par Gregor PERKO (2011 : 40) est pertinent, en ce sens qu'il explique au mieux la nature de ce marqueur, ainsi que le mécanisme de «l'émergence de nouveaux sens ».

L'espace tropologique ${ }^{6}$ du corpus analysé est ainsi représenté par des tropes composés d'un seul mot (14\%), des périphrases-énigmes $(21 \%)$ et des textes monophrastiques humoristiques $(9 \%)$. Certaines appellations métaphoriques et métonymiques ont été déjà incorporées dans le texte du présent article. Ajoutons d'autres exemples de désignations à valeur figurée: charbonettes ('jambes

\footnotetext{
${ }^{6}$ Cette notion a fait l'objet d'une de nos publications sur les particularités de la création des unités hors
} norme (RETINSKAYA, 2009). 
maigres'), croupillon ('partie postérieure de l'homme'), dos ('sorte de gilet sans manches'), housse du dos ('échine'), peau ou piau ('femme de rien'), tournant ('nuque'), avoir le cul berneux ('se sentir gêné'), grand soret ('personne maigre'), boudine d'agasse ('homme fier qui marche en bombant le torse'), faire la loupe ('bouder'), voir les acouplettes à quelqu'un ('être maigre') ...

\section{Distinction des unités régionales en fonction de leur vitalité}

En considérant la force vitale des régionalismes, l'analyse du vocabulaire en question peut se réaliser à partir d'une répartition du corpus en cinq groupes principaux, selon le classement élaboré par Fernand CARTON et Denise POULET (2008: 7-8). Nous les reproduisons en fonction de leur vitalité croissante et en les illustrant par des unités du corpus retenu :

1) les termes sans véritable correspondant français dont les occasions d'emploi sont rares ou ont disparu, comme poquelé ('personne présentant des cicatrices de varicelle');

2) les termes sans véritable équivalent français dont les occasions d'emploi sont assez fréquentes, comme loup ( $p$ )e ('lèvre inférieure épaisse et proéminente') ;

3) les termes vivants plus fréquemment employés que l'équivalent national: brelle ('cheveux raides');

4) les termes anciens hors d'usage employés plaisamment, telle l'expression les dents à crouchî un ramon (< les dents à croquer un balai) qui se dit de la physionomie de quelqu'un dont les incisives supérieures sont longues et basculées en avant ;

5) les régionalismes si répandus partout qu'ils ne sont plus sentis comme tels : boudette ('nombril').

L'étude de terrain effectuée dans le département des Ardennes $^{7}$ en janvier/février 2020 en présentiel et août/novembre 2020 à distance a montré qu'un nombre considérable des somatismes collectés $(74 \%)$ demeurent vivants et dans nombre de cas assez efficaces.

\section{En guise de conclusion}

La classification détaillée des éléments du corpus se rapportant au corps humain et la réalisation des enquêtes visant à apprécier la vitalité - et la pérennité - des ardennismes révèlent bien la richesse du vocabulaire régional dans sa capacité non seulement à nuancer la description du portrait physique d'un individu, mais aussi à servir de moyen pour se moquer de soi-même ou pour dévaloriser autrui. Le taux de

\footnotetext{
${ }^{7}$ Les enquêtes sont effectuées dans les communes de Lalobbe, La Neuville-lès-Wasigny, Librecy, Marlemont, Montmeillant, Neufmanil, Novion-Porcien, Perthes, Signy l'Abbaye. Je remercie vivement tous mes fidèles témoins et surtout Pascal RÉSER et Roger COPINE pour leur aide et leur volonté de sauvegarder le patrimoine.
} 
vitalité des vocables étudiés est assez élevé, car ils visent à désigner des concepts primordiaux et sont employés par les locuteurs de tout âge.

Cependant, il s'avère nécessaire de poursuivre et approfondir les recherches sur le terrain, notamment en élargissant notre champ d'investigation et en questionnant les habitants d'autres communes ardennaises, dans le but d'établir une cartographie web des centres d'intérêt inventoriés.

\section{BIBLIOGRAPHIE}

BÉSÈME-PIA Lise (2011), Patois ardennais. Le parler de mon village, Langres, Éditions Dominique Guéniot.

BOURCELOT Henri (1966), Atlas linguistique et ethnographique de la Champagne et de la Brie, I : Le temps - La terre, Paris, Éditions du CNRS.

BourCElot Henri, TAMINE Michel (éd. mise au point par -) (2012), Atlas linguistique et ethnographique de la Champagne et de la Brie, IV : Animaux sauvages - Activités humaines, Paris/Langres, Éditions du CTHS/Éditions Dominique Guéniot.

Braibant Charles (1933), Le Roi dort. Paris, Éditions Denoël et Steele.

CARTON Fernand \& POUlet Denise (2008), Le parler du Nord Pas-de-Calais, Paris, Christine Bonneton Éditeur.

CLERC Jean (1988-1989), « Un îlot linguistique des Hautes-Rivières (Ardennes) », Parlure Champagne-Ardenne, Les Cahiers de l'Institut Charles-Bruneau, 4-5, Charleville-Mézières, Institut Charles-Bruneau, p. 149-165.

Colin Jean (1957), Histoire de Neufmanil. Masnil. Vieux Manil. Neufmanil. « NunMani ». Dictionnaire - vocabulaire du "vieux» patois de "Neuf» Manil, Neufmanil.

CORDIER Jean-Pol (1975), Lexique du patois de Neufmanil et environs, Neufmanil [tapuscrit original].

CORDIER Jean-Pol (1988), Dictionnaire de patois ardennais : français-patois \& patois-français, Charleville-Mezières : Éditions de la Société des Écrivains Ardennais.

Glossaire des mots picards, termes et expressions encore en usage dans le Vermandois (1953-1954), in: Mémoires de la Fédération des Sociétés Savantes du département de l'Aisne, Laon, Baticle, 1, p. 57-66.

HUART Désiré (1988), Les patois de l'Est-Sedanais en particulier des cantons de Mouzon-Carignan et quelques villages limitrophes de la Meuse et de Belgique, Charleville-Mézières, Imprimerie ARDENN'OFFSET.

LaUby Mireille, TAmINE Michel (1987), Les sobriquets dans la Région de Signyle-Petit (Ardennes), Parlure Champagne-Ardenne, Les Cahiers de l'Institut Charles-Bruneau 3, Charleville-Mézières, Institut Charles-Bruneau, p. 33-45.

Patois de Guignicourt, Faissault et Champigneul-sur-Vence, renseignements divers (1929), (s.1.), 3 cahiers non paginés.

PERKO Gregor (2011), «Le rôle de la métonymie dans l'émergence du sens argotique », Registres de langue et argot(s). Lieux d'émergence, vecteurs de 
diffusion, in: BASTIAN Sabine, GoudaILlier Jean-Pierre (éd.), München, Éditions Martin Meidenbauer, p. 29-43.

PetitfauX Gemma (1962), Debout frères de misère, Paris, Éditions Alsatia.

RETINSKAYA Tatiana (2009), Les constituants de l'espace tropologique des argots français de métiers (sur l'exemple de l'argot des viticulteurs et des vignerons de Champagne), in : KACPRZAK Alicja, GOUDAILlIER Jean-Pierre (éd.), Standard et périphéries de la langue, Łódź - Łask, Oficyna Wydawnicza LEKSEM, p. 235-241.

RÉZEAU Pierre (éd.) (2001), Dictionnaire des régionalismes de France. Géographie et histoire d'un patrimoine linguistique, Bruxelles, Éditions De Boeck/Duculot. Rogissart Jean (1994), Les Romans rustiques, Charleville-Mézières, Éditions Terres Ardennaises.

TAMINE Michel (2006), Le parler des Ardennes, Paris, Christine Bonneton Éditeur. VAUCHELET Albert (1979), Tous les patois des Ardennes. Vieux langages et vieilles choses, Charleville-Mézières, Imprimerie SOPAIC - Savreux. 